\title{
Qualidade de Vida em Adultos com Deficiência Física
}

\author{
Quality of Life in Adults with Physical Disability
}

\author{
Marineia Crosara de Resende ${ }^{*}, a$ \& Valdiney Veloso Gouveia ${ }^{b}$ \\ ${ }^{a}$ Universidade Federal de Uberlândia, Uberlândia, Brasil \\ $\&{ }^{b}$ Universidade Federal da Paraíba, João Pessoa, Brasil
}

\begin{abstract}
Resumo
O objetivo dessa pesquisa foi investigar a qualidade de vida em adultos com deficiência física nos domínios: bem-estar subjetivo, competências comportamentais, qualidade de vida percebida e condições ambientais. Método: Participaram 25 pessoas, idade entre 32-69 anos. Responderam em entrevista aos instrumentos: (a) Ficha de Informações sócio-demográficas; (b) Ficha de Informações sobre a deficiência física; (c) Medida do Grau de Incapacidade; (d) Escala de sintomas físicos; (e) Medida de bem-estar subjetivo: composto por Escala de Vitalidade, Escala de Afetos Positivos e Escala de Satisfação com a Vida; (f) Escala de auto-eficácia física; (g) Questionário de Saúde Geral (QSG-12); (h) Escala de Percepção de Suporte Social. Resultados: Os participantes apresentam qualidade de vida positiva: relataram bem estar subjetivo, apresentaram senso de auto-eficácia no ponto médio, apresentam saúde mental positiva, com relação aos sintomas físicos, as queixas mais relatadas foram dores nos braços, pernas e articulações, relataram receber suporte social nos aspectos emocional e prático.

Palavras-chave: Deficiência física; Qualidade de vida; Psicologia; Gerontologia.
\end{abstract}

\begin{abstract}
The aim of this study was to investigate the quality of life in adults with physical disability in the following domains: subjective well-being, behavioral competence, perceived quality of life, and environmental conditions. Method: 25 participants aged between 32 and 69. They answered, in interview, the following instruments: (a) Socio-demographic Characteristics; (b) Physical Impairment Characteristics; (c) Degree of disability; (d) Physical Symptom Scale; (e) Subjective Well-being Measurement composed by: Vitality Scale, Positive Affect Scale, Life Satisfaction Scale; (f) Physical Self Efficacy Scale; (g) General Health Questionnaire (GHQ-12); (h) Perceived Social Support Scale. Results: The participants presented positive quality of life. They reported subjective well-being, showed self-efficacy in the average point, and presented positive mental health regarding physical symptoms. The most common complaints were related to pains in the arms, legs and joints. They reported to receive social support in emotional and practical aspects.
\end{abstract}

Keywords: Physical disability; Quality of life; Psychology; Gerontology.

Atualmente temos um número crescente de pessoas com deficiência física inseridas na luta por um mundo melhor e em busca de maior longevidade com qualidade de vida. A deficiência física ainda povoa a mente humana com pré-conceitos e tabus que vêm sendo perpetuados ao longo dos anos, tais como o mito da dependência e da improdutividade. Mas é importante ressaltar que ainda na presença de incapacidades, as pessoas podem equilibrar suas limitações com suas potencialidades e viver e envelhecer com qualidade de vida, mesmo tendo uma deficiência física (Resende, 2006).

"Endereço para correspondência: Universidade Federal de Uberlândia, Avenida Pará, 1720, Bloco 2C, Campus Umuarama, Uberlândia, MG, Brasil, CEP 3840o-902. Email: marineiaresende@gmail.com

A primeira autora agradece ao Conselho Nacional de Desenvolvimento Científico e Tecnológico (CNPQ) pela bolsa concedida de pós-doutorado júnior.
Neste sentido, segundo Neri (2004) considerando o aumento da atenção para a inclusão da pessoa com deficiência na sociedade, preconizado por vários organismos nacionais e internacionais, torna-se fundamental para os profissionais de saúde que trabalham com reabilitação, compreenderem como as pessoas com deficiência percebem sua qualidade de vida, se ajustam, vivem o dia-a-dia e envelhecem com uma deficiência (Resende, 2006).

Viver e envelhecer pressupõem alterações gradativas nos domínios físico, psicológico, social e ambiental. A idade do início das mudanças, o seu ritmo, os domínios atingidos e o resultado final sobre o comportamento dependem de características genéticas de cada indivíduo, em interação com o self, e também do seu estilo de vida, de condições passadas e atuais de apoio ao desenvolvimento, tais como nível de renda, educação e posição social. Sendo assim, a avaliação da qualidade de vida pode variar entre indivíduos vivendo em sociedades diferentes bem como entre grupos, minoritários, como o de pessoas 
com deficiência física, em uma mesma sociedade. Fatores objetivos e subjetivos estão em interação e, estão envolvidos em sua avaliação, critérios que são tidos como importantes e desejáveis dentro de um determinado grupo social e valores vigentes na sociedade, além de estarem sujeitos a mudanças no decorrer do tempo à medida que as sociedades se desenvolvem. Em qualquer sociedade e momento do ciclo de vida a qualidade de vida é um fenômeno de várias faces (Neri, 2001).

Neste contexto, um modelo amplamente aceito dentro da Gerontologia e que irá pautar esse estudo, é o de Lawton (1991). Levando em conta que a qualidade de vida é um construto multidimensional, neste modelo estão incluídos fatores objetivos - aqueles que podem ser verificados e observados mediante parâmetros externos - e fatores subjetivos - aqueles que pertencem ao âmbito da experiência privada e são baseados na avaliação que o indivíduo faz sobre as condições objetivas disponíveis (Neri, 2001).

O modelo de Lawton contempla quatro dimensões: a competência comportamental, a qualidade de vida percebida, as condições ambientais e o bem-estar subjetivo. Cada uma destas dimensões será apresentada separadamente, mas não se deve perder de vista o fato de que estas fazem parte de um todo que está interrelacionado e que qualquer mudança obtida em uma das dimensões irá inevitavelmente afetar as demais (Lawton, 1991).

A competência comportamental refere-se ao desempenho do indivíduo em seu cotidiano e de seu funcionamento quanto à saúde, funcionalidade física, cognição, comportamento social e utilização do tempo (Neri, 2001). Estas competências no seu dia-a-dia refletem em autonomia e independência, levando-se sempre em conta os aspectos objetivos, os subjetivos e os contextuais.

A competência comportamental pode ser avaliada em termos de auto-eficácia. De acordo com Bandura (1997), o senso de auto-eficácia corresponde às crenças de uma pessoa a respeito do grau e da extensão em que ela própria tem capacidade para ativar a motivação, resgatar a cognição e usar mecanismos de ação, que são necessários para exercer controle sobre tarefas específicas. Assim, a auto-eficácia é uma crença que leva a pessoa a comportar-se de certa forma baseada no que acredita. As crenças de auto-eficácia determinam em grande parte as decisões envolvendo as escolhas do curso de ação a ser tomado. A auto-eficácia percebida é um construto pertinente às percepções do indivíduo quanto a sua capacidade de conseguir com sucesso um determinado comportamento ou enfrentar uma situação.

A qualidade de vida percebida, segundo Neri (2001), refere-se à avaliação subjetiva da competência comportamental que o indivíduo faz mediante seus próprios critérios e parâmetros, da sua competência comportamental. Este é um domínio subjetivo paralelo a outro objetivo, a competência no cotidiano. Medidas tais como saúde percebida, alterações cognitivas percebidas e o senso de auto- eficácia, são exemplos relativos a este domínio. A autoeficácia, isto é, a convicção que o indivíduo tem de que é capaz de produzir os resultados esperados, é crítica nesta avaliação da competência comportamental. $\mathrm{O}$ mecanismo de comparação social - um dos mediadores da autoeficácia - é muito útil para promover um senso positivo de si mesmo uma vez que essa comparação é feita tendo como parâmetro outro indivíduo, especialmente os da mesma coorte, em piores condições.

O domínio condições ambientais é referente aos ambientes tanto no sentido espacial como as cidades, as habitações e as instituições, bem como no sentido social abrangendo as políticas públicas, a formação de recursos humanos e índices de desenvolvimento social (M. E. C. Siqueira, 2003) e ao manejo utilizado para lidar com este ambiente, que pode ser mais fácil quando a pessoa conta com suporte social. Estes ambientes exercem certas pressões sobre as pessoas que procuram um equilíbrio se adaptando ou mudando ativamente o espaço (Wahl, 1999). Este domínio tem relação direta com a competência comportamental uma vez que atua como facilitador ou como obstáculo no desempenho de atividades do cotidiano. O ambiente deve ser compatível às competências da pessoa, compensando perdas, prevenindo acidentes, fornecendo estímulos e desafios e promovendo autonomia e independência (Neri, 2001).

Gignac e Cott (1998) afirmam que a deficiência é um fenômeno multidimensional e que por esse motivo, deve ser reconhecido que as pessoas apresentam dificuldades físicas em diferentes domínios. Os autores apontam sete desses domínios: (a) cuidados pessoais, incluindo atividades básicas de vida diária, como por exemplo, comer, tomar banho, vestir-se, usar o banheiro; (b) tarefas domésticas, mobilidade pessoal em casa, incluindo preparar refeições, limpar a casa, providenciar as compras da casa, entre outras atividades instrumentais de vida diária; (c) mobilidade em casa, fazer transferência da cama e da cadeira de rodas, andar sozinho pela casa, levantar-se, subir e descer escadas; (d) mobilidade na comunidade, participar de atividades comunitárias relacionadas a sair de casa sozinho, entrar e sair do carro, usar outros meios de transporte como ônibus e metrôs, andar pela cidade, em avenidas principais, prédios e áreas abertas; (e) atividades prazerosas, incluindo atividade de lazer, passatempos, socialização com outras pessoas, entretenimento em casa e viajar por prazer. Essas atividades parecem ter importantes implicações para o bem estar dos indivíduos e podem estar relacionadas ao senso de dependência e independência; (f) cuidar de outras pessoas, como crianças e outros parentes; (g) ter um emprego.

Segundo os mesmos autores, considerar esses domínios permite entender melhor o impacto que as deficiências exercem sobre a vida do indivíduo e sobre sua percepção de dependência ou independência: (a) algumas variáveis estão relacionadas a entender se a pessoa precisa ou não de assistência, (b) outras estão relacionadas a 
se a pessoa receberá ou não assistência, e (c) outras ainda, relacionam-se à percepção da pessoa sobre sua autonomia.

Os ajustes entre o nível de competência de uma pessoa e as demandas do ambiente afetam o funcionamento e indicam saúde e funcionalidade física e mental (Lawton, 1991). De acordo com M. M. M. Siqueira (2008), vários estudiosos passaram a investigar com maior ênfase a interface ambiente social e saúde a partir do estudo de Berkman e Syme (1979), procurando identificar tipos de relacionamentos benéficos à saúde. Portanto, o suporte social é considerado capaz de gerar efeitos benéficos tanto para a saúde física como mental, guardando uma estreita relação com bem-estar. Trata-se de um conceito multidimensional que se refere aos recursos materiais e também psicológicos aos quais as pessoas têm acesso através de suas redes sociais (M. M. M. Siqueira, 2008).

As redes sociais são definidas como "teias" de relações sociais que circundam o indivíduo bem como suas características - por exemplo, disponibilidade e freqüência de contato com amigos e parentes - ou com grupos de pessoas com quem há contato ou alguma forma de participação social - por exemplo, grupos religiosos, associações sindicais (G. R. B. Andrade, 2001). Griep, Chor, Faerstein e Lopes (2003) afirmam que apoio social refere-se a um sistema de relações formais e informais pelo qual os indivíduos recebem ajuda emocional, material e/ ou de informação para enfrentarem situações geradoras de tensão emocional. Trata-se de um processo recíproco que gera efeitos positivos tanto para o sujeito que recebe como também para quem oferece $\mathrm{o}$ apoio, permitindo que ambos tenham mais sentido de controle sobre suas vidas. Nesse processo se aprende que as pessoas necessitam umas das outras.

Os contatos sociais permitem engajamento social que também é uma forma de se vivenciar o desenvolvimento na idade adulta, de maneira bem sucedida. $\mathrm{O}$ apoio social deve ser compreendido como uma experiência pessoal e subjetiva que leva a um maior senso de satisfação com a vida. Silva et al. (2003) sugerem a existência de uma relação entre apoio social e uma variedade de medidas dependentes: saúde, adaptação psicológica, percepção de bem-estar, redução do mal estar, longevidade e mortalidade, satisfação com a vida, entre outros.

O bem-estar subjetivo é um construto referente à avaliação que a própria pessoa faz sobre sua vida e, este tipo de avaliação pode ser feita mediante um componente cognitivo como julgamento sobre a satisfação com a vida como um todo ou referenciada a domínios específicos ou mediante um componente afetivo no qual estão incluídos estados agradáveis e desagradáveis e a busca de equilíbrio entre eles. Neste domínio está refletida a avaliação que o indivíduo faz dos domínios anteriores. $\mathrm{O}$ âmbito do bem-estar subjetivo tem interessado bastante aos psicólogos e segundo Diener (2000) é uma das áreas de análise da Psicologia Positiva. Pesquisas no mundo indicam que a felicidade e a satisfação com a vida são muitos im- portantes e que os indivíduos pensam a respeito delas freqüentemente.

De acordo com Lawton (1991) é preciso desenvolver competências de vida diária, relativas ao auto-cuidado e ao manejo de atividades instrumentais rotineiras, necessárias à sobrevivência, por exemplo, utilizar transporte $\mathrm{e}$ fazer compras. E também inclui eventos sociais e de lazer escolhidos pela pessoa, bem como atividades instrumentais que assegurem o contato da pessoa com o mundo externo e com o self.

É relevante pesquisar sobre esses temas para entender como os mecanismos internos de auto-regulação, as condições ambientais, a qualidade de vida percebida e o bemestar subjetivo interagem entre si, podendo contribuir para que as pessoas com deficiência física alcancem um envelhecimento com qualidade de vida.

Neste sentido esta pesquisa pretende investigar a qualidade de vida em pessoas com deficiência física nos seguintes domínios: bem-estar subjetivo - estado de ânimo, vitalidade e satisfação com a vida; competências comportamentais - grau de incapacidade, manejo da incapacidade, crença de auto eficácia física; qualidade de vida percebida e condições ambientais - percepção de suporte social, em pessoas com deficiência física.

\section{Método}

\section{Participantes}

Esta pesquisa foi realizada com uma amostra de conveniência retirada do universo de participantes de Conselho Nacional dos Centros de Vida Independente CVIBrasil. Os critérios de inclusão para participação foram: ter idade acima de 25 anos; ter deficiência física e utilizar auxílio ortopédico (cadeira de rodas, muleta, órtese ou andador) para realizar as atividades básicas e instrumentais de vida diária; ser alfabetizado e ter sido indicado pelo Presidente ou demais integrantes do CVI - Brasil, por ser considerado um líder no movimento social pela causa das pessoas com deficiência física.

Participaram 25 pessoas, com idade entre 32 e 69 anos $(M=48$ anos $\pm 8,9)$. A maioria dos participantes é do sexo feminino (64,0\%); solteiro $(56,0 \%)$, casado $(28,0 \%)$, viúvo $(8,0 \%)$, separado $(8,0 \%)$; cursou pós-graduação $(60,0 \%)$, ensino superior $(24,0 \%)$, ensino médio $(12,0 \%)$ e ensino básico $(4,0 \%) ; 84,0 \%$ trabalha; $32,0 \%$ é aposentado e, entre eles, $87,5 \%$ por invalidez; $12,5 \%$ por tempo de serviço; sendo que $76,0 \%$ vive na região sudeste, $16,0 \%$ no nordeste e $8,0 \%$ na região sul.

\section{Instrumentos}

Ficha de Informações Sócio-Demográficas. Questionário para coleta de dados sobre idade, gênero, renda mensal, profissão, ocupação, estado civil e tipo de arranjo domiciliar.

Ficha de Informações sobre a Deficiência Física. Questionário para coleta de dados sobre a deficiência: nome e origem (congênita ou adquirida); se adquirida, idade e 
motivo da aquisição da deficiência; utilização de auxílio ortopédico e de outros tipos de apoio.

Medida de Bem-Estar Subjetivo. Composta pelos seguintes instrumentos: (a) Escala de Vitalidade (Chaves, 2003): avalia o grau em que o participante tem se sentido em termos de vigor físico, mental e alerta, nos últimos dias. Compõe-se de sete itens (por exemplo, tenho energia e disposição; sinto-me vivo e cheio de vitalidade), cujas respostas devem ser dadas em uma escala de sete pontos, indo de $1=$ Nada Verdadeiro a 7 = Totalmente Verdadeiro. (b) Escala de Afetos Positivos e Negativos (Chaves, 2003): avalia estado de ânimo, formada por nove adjetivos, sendo 5 positivos: feliz, alegre, satisfeito, divertido e otimista; e 5 negativos: deprimido, preocupado, frustrado, raivoso e infeliz. O instrumento avalia quanto o participante tem experienciado cada uma dessas emoções nos últimos dias. Os itens são respondidos numa escala de sete pontos, variando de 1 = Nada a $7=$ Extremamente. (c) Escala de Satisfação com a Vida (Chaves, 2003): compõe-se de cinco itens (por exemplo, estou satisfeito com minha vida; as condições da minha vida são excelentes), respondidos em escala tipo Likert, indo de $1=$ Discordo Totalmente a 7 $=$ Concordo Totalmente.

Medida de Competências Comportamentais. Composto pelos seguintes instrumentos: (a) Medida do Grau de Incapacidade (Resende, 2006) - pergunta-se aos participantes o grau de dificuldade que eles apresentam para desempenhar 30 atividades, divididas em cinco domínios: cuidados pessoais, mobilidade dentro de casa, mobilidade na comunidade; atividades domésticas; atividades prazerosas. As respostas são pontuadas em: 0 (nenhuma dificuldade), 1 (alguma dificuldade); 2 (muita dificuldade); 3 (incapaz de realizá-la).

(b) Escala de Auto-eficácia Física (Leão, 2003). Trata-se de instrumento com 22 itens que avaliam as capacidades físicas para as quais a respondente indica o grau de confiança nas suas capacidades físicas. A avaliação é feita numa escala Likert de cinco pontos com a classificação que varia de "concordo totalmente" a "discordo totalmente". A escala abrange duas variáveis: habilidade ou capacidade física percebida (10 itens) e confiança na auto-apresentação física - auto-imagem (12 itens).

Medida de Qualidade de Vida Percebida. Composta pelos seguintes instrumentos: (a) Escala de Sintomas Físicos (Wang, 2002): contendo uma lista de queixas somáticas categorizadas por aparelhos do organismo humano, tendo em vista que queixas físicas podem ser marcadores para mensuração do nível de estresse psíquico dos sujeitos; (b) Questionário de Saúde GeralQSG-12 (Gouveia et al., 2003): composto de 12 itens (por exemplo: Você tem se sentido pouco feliz e deprimido?; Você tem perdido a confiança em si mesmo?). Cada item respondido, avalia quanto a pessoa tem experimentado os sintomas descritos, sendo suas respostas dadas em uma escala de quatro pontos. No caso dos itens que negam a saúde mental (por exemplo: Suas preocupações lhe têm feito perder muito sono?; Tem se sentido pouco feliz e deprimido?), as alternativas de resposta variam de 1 (Absolutamente, não) a 4 (Muito mais que de costume); no caso dos itens afirmativos (por exemplo: Tem se sentido capaz de tomar decisões?; Tem podido concentrar-se bem no que faz?), as respostas foram de 1 (Mais que de costume) a 4 (Muito menos que de costume). Os itens negativos foram invertidos, de modo que a maior pontuação total nesta medida indica melhor nível de saúde mental.

Medida de Condições Ambientais. Composta pela Escala de Percepção de Suporte Social (M. M. M. Siqueira, 2008) - contém 29 itens, divididos em 2 dimensões: Suporte prático e Suporte emocional. As respostas devem ser dadas numa escala de quatro pontos $(1=$ nunca; $2=$ poucas vezes; $3=$ muitas vezes; $4=$ sempre) com a qual os participantes informaram a frequencia com que percebem a possibilidade de poder contar com o apoio de alguém.

\section{Resultados}

Para descrever o perfil da amostra segundo as variáveis em estudo, foram feitas tabelas de freqüência das variáveis categóricas e medidas de posição e dispersão.

\section{Bem-Estar Subjetivo}

Os participantes dessa pesquisa relatam bem estar subjetivo positivo indicado por satisfação com a vida (média 5,$08 ; D P=1,10$ ), afetos positivos (média 5,$00 ; D P=1,13$ ), afetos negativos (média 3,$02 ; D P=0,85$ ) e vitalidade (média 5,$12 ; D P=0,82)$.

Auto-Relatos sobre Competências Comportamentais Grau de Incapacidade, Manejo da Incapacidade; Crença de Auto-Eficácia Física

A maioria acredita que o grau de sua deficiência é moderado $(64,0 \%)$ e o restante da amostra avalia que sua deficiência é severa $(36,0 \%)$; possuem uma deficiência congênita $(34,0 \%)$ ou adquirida $(76,0 \%)$ em função de doença $(44,0 \%)$, acidentes $(36,0 \%)$ ou outros $(20,0 \%)$.

Todos os participantes relataram contar com ajuda de alguém $(100,0 \%)$. As fontes de ajuda não eram exclusivas e provinham principalmente da família $(84,0 \%)$, de empregados $(68,0 \%)$, amigos (48,0\%) e cônjuge (40,0\%).

\section{Grau de Incapacidade para as Atividades Cotidianas}

Foi calculada a média das pontuações apresentadas pela amostra nos itens da escala que avaliava o grau de dificuldade para desempenhar atividades cotidianas. Todas as pessoas deste estudo relataram ter alguma necessidade de ajuda de outras pessoas ou de algum dispositivo ou equipamento. Na Tabela 1 pode ser visto o grau de dificuldade por domínio. 
Tabela 1

Grau de Incapacidade por Categoria de Atividades

\begin{tabular}{lrc}
\hline \multicolumn{1}{c}{ Variável } & Média & $D P$ \\
\hline Cuidados pessoais & 1,05 & 0,96 \\
Mobilidade dentro de casa & 1,74 & 0,56 \\
Mobilidade na comunidade & 1,56 & 0,81 \\
Atividades domésticas & 1,46 & 0,63 \\
Atividades prazerosas & 0,73 & 0,39 \\
\hline
\end{tabular}

\section{Senso de Auto-Eficácia}

As pessoas com deficiência física desta pesquisa apresentaram senso de auto-eficácia no ponto médio, ou seja, suas crenças nas suas habilidades e capacidades não são nem positivas e nem negativas (média 3,12; $D P=0,26$ ), na Capacidade Física Percebida (média 3,17; $D P=0,47$ ) e na Auto-imagem (média 3,67; $D P=0,27$ ).

Qualidade de Vida Percebida: Saúde Mental (Ausência de Depressão e Ansiedade); Sintomas Físicos Percebidos

O questionário de saúde geral, a média foi de 1,85; $D P=0,42$, no escore de depressão do QSG-12, a média foi de $1,68(D P=0,40)$. No escore de ansiedade do QSG12 , a média foi de $2,19(D P=0,56)$; o que indica que os sujeitos apresentam saúde mental positiva, já que, quanto mais próximo de 1 , melhor a saúde mental, quanto mais próximo de 4, maior o distresse.

Com relação aos sintomas físicos foi verificado que as queixas mais relatadas são: Dores - dor nos braços, pernas e articulações $(72,0 \%)$, dor nas costas $(64,0 \%)$ e dor lombar $(52,0 \%)$.

Com relação ao número de sintomas físicos, as pessoas relataram apresentar em média $1,81(D P=0,92)$ queixas.

\section{Condições Ambientais: Percepção de Suporte Social}

Com relação à percepção de suporte social, os participantes relataram perceber suporte social nos aspectos: suporte emocional (média 3,10; $D P=0,63$ ) e suporte prático (média 2,$87 ; D P=0,59$ ).

\section{Qualidade de Vida em Pessoas com Deficiência Física}

Levando-se em consideração as variáveis propostas e seus respectivos resultados - (a) Competência comportamental: a incapacidade funcional existe em função da própria deficiência e as crenças de auto-eficácia foram neutras; (b) Qualidade de vida percebida: saúde mental foi positiva (ausência de depressão e ansiedade) e os sintomas físicos percebidos são em pequeno número; (c) Bem-estar subjetivo: estado de ânimo, vitalidade e satisfação com a vida, também positivos; (d) Condições ambientais: percepção de suporte social positiva, tanto nos aspectos emocionais como práticos - pode-se considerar que as pessoas com deficiência física apresentam qualidade de vida positiva.

\section{Discussão}

Nesta pesquisa investigou-se a qualidade de vida em pessoas com deficiência física, considerando o modelo de Lawton. Os participantes relatam bem-estar subjetivo positivo indicado por satisfação com a vida, afetos positivos e vitalidade. Segundo Guedea et al. (2006) o bemestar subjetivo, além de ser um indicador de saúde mental, tem sido descrito como sinônimo de felicidade, ajuste e integração social. Diener, Suh, Lucas e Smith (1999) relatam que as pesquisas têm indicado que a satisfação com a vida não diminui com a idade, apesar do declínio associado à idade em recursos como saúde, situação conjugal e renda, mas que a intensidade das experiências afetivas diminui ao longo do envelhecimento.

Resende, Cunha, Silva e Souza (2007), em pesquisa com 90 pessoas com idade entre 25 e 85 anos, encontraram que os participantes apresentaram elevado grau de satisfação com a vida e expectativa positiva com relação à vida futura. As pessoas que apresentaram maior satisfação com a vida foram as que recebiam mais suporte afetivo.

As pessoas desse estudo relataram incapacidade para o desempenho de atividades cotidianas e, na avaliação subjetiva de como percebem sua deficiência, a maioria afirmou que sua deficiência é moderada, gerando um grau de dependência que determina necessidade de ajuda de outras pessoas, principalmente da família. A família tem sido relatada na literatura como sendo a principal fonte de apoio ao longo da vida (Antonucci \& Jackson, 1997; Nogueira, 2001), desde o nascimento da criança até a morte. A dinâmica do grupo familiar é muito poderosa no desenvolvimento da criança, contribuindo para aumentar a competência adaptativa, particularmente de crianças com deficiência física. A família auxilia na construção do autoconceito da criança, de suas conviç̧ões, valores e atitudes sobre si mesma, contribuindo para o desenvolvimento do amor próprio e do senso de aceitação social à medida que ficam mais velhas (Resende \& Leão, 2008). Ao longo do desenvolvimento, família e amigos intercalam papéis de importância na vida da pessoa, sempre relacionados à manutenção do bem estar e da saúde, à confirmação do self e à aceitação social.

As pessoas com deficiência física dessa pesquisa apresentaram senso de auto-eficácia no ponto médio, ou seja, suas crenças nas suas habilidades e capacidades não são nem positivas e nem negativas. $\mathrm{O}$ fato de as crenças de auto-eficácia terem sido "neutras", já que o instrumento ${ }^{1}$ utilizado avalia auto-eficácia com relação à capacidade física, pode indicar que essas pessoas estão conscientes de sua condição, não negam a própria realidade, o que pode ser fator de adaptação que as favorece. Se apresentam algum grau de dependência, sabem que continuarão

${ }^{1}$ É importante ressaltar, que o instrumento utilizado talvez não seja o mais adequado para avaliar pessoas com deficiência física. 
dependendo independentemente de acreditarem no seu potencial, e que, em alguns casos essa dependência poderá aumentar quando chegarem à velhice.

Os sistemas de crenças e de atitudes têm função adaptativa e segundo Rokeach (1981), eles ajudam os indivíduos a definir e compreender o mundo e a si mesmos, formar atitudes e se posicionar diante das situações vividas no dia-a-dia. As crenças de auto-eficácia desempenham um papel importante nas relações dos indivíduos com o mundo porque refletem as suas avaliações quanto à própria capacidade de comportar-se de forma adequada e produtiva em situações específicas; influenciam o esforço que vão imprimir em suas ações, sua perseverança frente às dificuldades, suas auto-avaliações, suas avaliações sobre o mundo externo e suas experiências emocionais (Bandura, 1986).

As pessoas com crenças auto-eficazes mais intensas são também as mais resilientes ao confrontarem-se com adversidades. Bandura (1997) afirma que quando as pessoas têm crenças positivas de auto-eficácia, no sentido de que acreditam que possam exercer certo controle sobre suas vidas, incluindo aqui as adversidades, se adaptam melhor a elas. No entanto, uma percepção inadequada de auto-eficácia, poderia gerar sentimentos de que apesar dos esforços empreendidos diante de uma nova realidade, nada do que for feito, irá adiantar (Vinaccia, Contreras, Londoño, Cadena, \& Anaya, 2005).

Os participantes dessa pesquisa apresentam saúde mental positiva, embora haja estudos como os de Turner, Lloyd e Taylor (2006) que afirmam haver um considerável número de informações sobre deficiência física e problemas mentais, no sentido negativo. Esses autores destacam que a maioria é inconsistente, seja pelo tamanho da amostra ou pela metodologia utilizada. Já Hermansson, Timpka e Thyberg (2002) afirmam que não existe relação entre o grau de deficiência física e saúde mental, mas que existe relação entre dor crônica e saúde mental, que apesar de coexistirem não se sabe a relação causal entre elas. Pirpiris et al. (2006) também não encontraram correlação positiva entre função física e saúde mental, considerando o bem-estar psicossocial.

Com relação aos sintomas físicos houve poucas queixas, sendo as mais relatadas relacionadas à dores nos braços, pernas e articulações, nas costas e lombar. As pessoas com deficiência física muitas vezes exigem mais de seus membros para a realiz

ação das atividades de vida diária para garantir a própria independência, quer seja no ato da locomoção, da transferência, dos autocuidados e outros. O maior esforço justificaria a maior ocorrência de dor nos membros. Segundo Gianini, Chamlian e Arakaki (2006), alguns usuários de cadeira de rodas experimentam dor em membros superiores que interfere nas atividades essenciais de vida diária, como ao fazer a propulsão da própria cadeira, dirigir, vestir-se e realizar transferências. Os autores relatam que a incidência de dor crônica, em membros superiores, pode limitar a função e interferir na inde- pendência e ser um sério problema para esta população, levando a uma significativa limitação funcional, afetando diretamente as atividades de vida diária, atividades profissionais e a realização de atividades físicas esportivas, quer seja a nível recreativo ou profissional.

É importante ressaltar que dores constantes podem tornar-se crônicas. A dor crônica é descrita como uma dor que pode durar meses, anos ou a vida toda, podendo haver ocorrências intermitentes que se caracterizam por períodos de dor, intercalados com intervalos isentos de dor, sendo que pode ser constante, persistir e piorar progressivamente, mesmo estando em tratamento (F. A. Andrade, Pereira, \& Sousa, 2006), ocasionando outros problemas.

Com relação à percepção de suporte social, os participantes relataram perceber suporte social, emocional e prático. Krause (2007) relata que quando os membros sociais das redes de relações fornecem auxílio, estão oferecendo muito mais que meramente oferecer suporte emocional e informativo. Receber suporte auxilia as pessoas a ter senso de pertencimento, a sentirem-se parte do grupo, identificando-se com o mesmo, encontrando motivação; traz senso de propósito e significado na vida.

Para Doeglas et al. (1994), o suporte social tem duas funções importantes: sustentar a saúde, que tem um efeito direto no bem-estar do indivíduo e reduzir o estresse, atuando como fator de proteção, frente a eventos adversos. O apoio social é uma variável importante, facilitadora de resultados positivos para o bem-estar subjetivo. É um conceito multidimensional, que engloba recursos materiais e psicológicos que são acessados através das redes sociais das pessoas (M. M. M. Siqueira, 2008). Indivíduos que têm altos níveis de apoio social e estão satisfeitos com a qualidade de sua rede de relações têm maior senso de bem-estar (Caprara \& Steca, 2005).

Lovden, Ghisletta e Lindenberger (2005) afirmam que pessoas engajadas, que participam de atividades diversas, tendem a ter melhor desempenho no funcionamento cognitivo. Nesse processo o suporte social parece ser fundamental para a emergência do bem-estar subjetivo e da qualidade de vida.

De acordo com Liptak (2008), pesquisas com adultos com deficiência, em geral, têm indicado que um ambiente suportivo (estratégias familiares de enfrentamento, apoio social forte) e capacidade individual de adaptação (motivação, força interior) são determinantes para a qualidade de vida, e as características de incapacidade são menos importantes.

Portanto, pode-se considerar que as pessoas com deficiência física apresentam qualidade de vida positiva, embora nessa pesquisa a amostra tenha sido apenas com pessoas engajadas socialmente, líderes de movimento social. Para Minayo, Hartz e Buss (2000), qualidade de vida é uma noção que, de acordo com a própria cultura, crenças, valores e expectativas, pressupõe a capacidade de unificar todos os elementos essenciais para o bemestar e a satisfação do indivíduo em uma sociedade. 
Envelhecer com uma deficiência física é um processo que exige competência adaptativa, capacidade para responder com resiliência aos eventos que surgem na vida e para ajustar-se aos desafios acarretados pela deficiência. Implica em ter discernimento de prioridades, manter relações sociais positivas, lutar por direitos, encontrar equilíbrio para lidar com as adversidades físicas e atitudinais impostas por uma sociedade ainda despreparada para a diversidade humana, para respeitar as diferenças e as particularidades de cada pessoa (Resende, 2006).

\section{Referências}

Andrade, G. R. B. (2001). Grupo de apoio social no hospital: O caso do lutando para viver. Dissertação de Mestrado nãopublicada, Fundação Osvaldo Cruz, Rio de Janeiro, RJ.

Andrade, F. A., Pereira, L. V., \& Sousa, F. A. E. F. (2006). Mensuração da dor no idoso: Uma revisão. Revista LatinoAmericana de Enfermagem, 14(2), 271-276.

Antonucci, T. C., \& Jackson, J. S. (1997). Apoyo social, eficácia interpersonal y salud: una perspectiva del transcurso de la vida. In L. L. Carstensen \& B. A. Edelstein (Eds.), Gerontología clínica: intervención psicológica y social (pp. 129-148). Barcelona, España: Martínez Roca.

Bandura, A. (1986). Social foundations of thought and action: A social cognitive. Englewood Cliffs, NJ: Prentice Hall.

Bandura, A. (1997). Self-efficacy: The exercise of control. New York: Freeman.

Berkman, L. F., \& Syme, L. S. (1979). Social networks, host resistance, and mortality: A nine-year follow-up study of Alameda County residents. American Journal of Epidemiology, 109, 186-204.

Caprara, G. V., \& Steca, P. (2005). Affective and social selfregulatory efficacy beliefs as determinants of positive thinking and happiness. European Psychologist, 10(4), 275-286.

Chaves, S. S. S. (2003). Valores como preditores do bem-estar subjetivo. Dissertação de Mestrado não-publicada, Universidade Federal da Paraíba, João Pessoa, PB.

Diener, E. (2000). Subjective well-being: The science of happiness and a proposal for a national index. American Psychologist, 55(1), 34-43.

Diener, E., Suh, E. N., Lucas, R. E., \& Smith, H. L. (1999). Subjective well-being: Three decades of progress. Psychological Bulletin, 125, 276-302.

Doeglas, D., Suurmeijer, T., Krol, B., Sanderman, R., Rijswijk, M. V., \& Leeuwen, M. V. (1994). Social support, social disability, and psychological well-being in rheumatoid arthritis. Arthritis Care and Research, 7, 10-15.

Gianini, P. E. S., Chamlian, T. R., \& Arakaki, J. C. (2006). Dor no ombro em pacientes com lesão medular. Acta Ortopédica Brasileira, 14(1), 44-47.

Gignac, M. A. M., \& Cott, C. (1998). A conceptual model of independence and dependence for adults with chronic physical illness and disability. Social Science and Medicine, 47(6), 793-753.

Gouveia, V. V., Chaves, S. S. S., Oliveira, I. C. P., Dias, M. R., Gouveia, R. S. V., \& Andrade, P. R. (2003). A utilização do QSG-12 na população geral: Estudo de sua validade de construto. Psicologia: Teoria e Pesquisa, 19, 241-248.

Griep, R. H., Chor, D., Faerstein, E., \& Lopes, C. S. (2003). Apoio social: Confiabilidade teste-reteste de escala no Estudo Pró-Saúde. Cadernos de Saúde Pública, 19(2), 625-634.
Guedea, M. T. D., Albuquerque, F. J. B., Troccoli, B. T., Noriega, J. A. V., Seabra, M. A. B., \& Guedea, R. L. D. (2006). Relação do bem-estar subjetivo, estratégias de enfrentamento e apoio social em idosos. Psicologia: Reflexão e Crítica, 19(2), 301-308.

Hermansson, A-C., Timpka, T., \& Thyberg, M. (2002). The mental health of war-wounded refugees: An 8-year followup. The Journal of Nervous and Mental Disease, 190(6), 374-380.

Krause, N. (2007). Longitudinal study of social support and meaning in life. Psychology and Aging, 22(3), 456-469.

Lawton, M. P. (1991). A multidimensional view of quality of life in frail elderly. In J. E. Birren, J. E. Lubben, J. C. Rowe, $\&$ D. E. Deutchmann (Eds.), The concept and measurement of quality of life in the frail elderly (pp. 3-27). San Diego, CA: Academic Press.

Leão, R., Jr. (2003). Participação em hidroginástica, crenças de auto-eficácia e satisfação com a vida em mulheres de 50 a 70 anos. Dissertação de Mestrado não-publicada, Universidade Estadual de Campinas, SP.

Liptak, G. S. (2008). Health and well being of adults with cerebral palsy. Current Opinion in Neurology, 21, 136-142.

Lovden, M., Ghisletta, P., \& Lindenberger, U. (2005). Social participation attenuates decline in perceptual speed in old and very old age. Psychology and Aging, 20(3), 423-434.

Minayo, M. C. S., Hartz, Z. M. A., \& Buss, P. M. (2000). Qualidade de vida e saúde: Um debate necessário. Revista Ciência \& Saúde Coletiva, 5(1), 7-18.

Neri, A. L. (2001). Velhice e qualidade de vida na mulher. In A. L. Neri (Ed.), Desenvolvimento e envelhecimento (pp. 161200). Campinas, SP: Papirus.

Neri, A. L. (2004). O que a Psicologia tem a oferecer ao estudo e à intervenção no campo do envelhecimento no Brasil, hoje. In A. L. Neri \& M. S. Yassuda, Velhice bem-sucedida (pp. 13-27). Campinas, SP: Papirus.

Nogueira, E. J. (2001). Rede de relações sociais: Um estudo transversal com homens e mulheres pertencentes a três grupos etários. Tese de Doutorado não-publicada, Universidade Estadual de Campinas, SP.

Pirpiris, M., Gates, P. E., McCarthy, J. J., D’Astous, J. D., Tylkowski, C., Sanders J. O., et al. (2006). Function and wellbeing in ambulatory cerebral palsy. Journal of Pediatric Orthopaedics, 26, 119-124.

Resende, M. C. (2006). Ajustamento psicológico, perspectiva de envelhecimento pessoal e satisfação com a vida em adultos e idosos com deficiencia física. Tese de Doutorado nãopublicada, Universidade Estadual de Campinas, SP.

Resende, M. C., Cunha, C. P. B, Silva, A. P., \& Souza, S. J. (2007). Rede de relações e satisfação com a vida em pessoas com amputação de membros. Ciências \& Cognição, 10, 164177.

Resende, M. C., \& Leão, R., Jr. (2008). Respeito pelo lar e pela família. In A. P. C. Resende \& F. M. P. Vital (Eds.), A convenção sobre os direitos das pessoas com deficiência comentada (pp. 78-81). Brasília, DF: Secretaria Especial dos Direitos Humanos.

Rokeach, M. (1981). Crenças, atitudes e valores: Uma teoria de organização e mudança. Rio de Janeiro, RJ: Interciência.

Silva, I., Pais-Ribeiro, J., Cardoso, H., Ramos, H., Carvalhosa, S. C., Dias, S., et al. (2003). Efeitos do apoio social na qualidade de vida, controle metabólico e desenvolvimento de complicações crônicas em indivíduos com diabetes. Psicologia, Saúde e Doenças, 4(1), 21-32. 
Siqueira, M. E. C. (2003). Políticas Públicas e qualidade de vida na velhice. In $3^{\circ}$ Congresso Paulista de Geriatria e Gerontologia [CD-ROM]. Santos, SP: Sociedade Brasileira de Geriatria e Gerontologia.

Siqueira, M. M. M. (2008). Construção e validação da Escala de Percepção de Suporte Social. Psicologia em Estudo, 13(2), 381-388.

Turner, R. J., Lloyd, D. A., \& Taylor, J. (2006). Physical disability and mental health: An epidemiology of psychiatric and substance disorders. Rehabilitation Psychology, 51(3), 214-223.

Vinaccia, S., Contreras, F., Londoño, L. M. R., Cadena, J., \& Anaya, J. M. (2005). Autoeficacia, desesperanza aprendida e incapacidad funcional en pacientes con diagnóstico de artritis reumatoide. International Journal of Clinical and Health Psychology, 5(1), 129-142.

Wahl, H. W. (1999). A competência no cotidiano: Um construto buscando uma identidade. Estudos Interdisciplinares sobre Envelhecimento, 2, 103-120.

Wang, Y. P. (2002). Manifestações psicopatológicas nãopsicóticas em uma amostra da comunidade chinesa da cidade de São Paulo. Tese de Doutorado não-publicada, Universidade Estadual de São Paulo, SP. 